\title{
White Matter Brain Development after Exposure to Circulating Cell-Free Hemoglobin and Hyperoxia in a Rat Pup Model
}

\author{
Åsa Jungner ${ }^{a}$ Suvi Vallius Kvist ${ }^{b}$ Olga Romantsik ${ }^{a} \quad$ Matteo Bruschettini $^{b}$ \\ Claes Ekström $^{b}$ Ivo Bendix ${ }^{c}$ Josephine Herz ${ }^{c}$ Ursula Felderhoff-Mueser ${ }^{c}$ \\ Adnan Bibic ${ }^{d}$ René In'T Zandt ${ }^{d}$ Magnus Gram ${ }^{b}$ David Ley ${ }^{a}$ \\ aDepartment of Clinical Sciences Lund, Pediatric Surgery and Neonatal Care, Skane University Hospital, Lund \\ University, Lund, Sweden; ${ }^{b}$ Department of Clinical Sciences Lund, Pediatrics, Lund University, Lund, Sweden; \\ 'Department of Pediatrics I, Neonatology and Experimental Perinatal Neuroscience, University Hospital Essen,

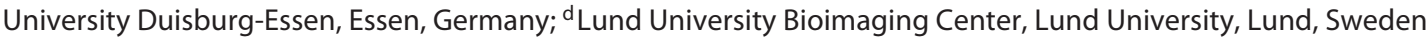

\section{Keywords}

White matter · Oligodendrocyte · Cell-free hemoglobin ·

Hyperoxia $\cdot$ Cardiopulmonary bypass

\begin{abstract}
Neonates born with critical congenital heart defects are at risk of diffuse white matter injuries and neurodevelopmental impairments. This study aimed to determine the impact of circulating cell-free hemoglobin and hyperoxia, both present during cardiopulmonary bypass circulation, on white matter brain development. Postnatal day 6 rat pups were injected intraperitoneally with cell-free $\mathrm{Hb}$ or vehicle and exposed to hyperoxia $\left(f_{i} \mathrm{O}_{2}=0.8\right)$ or normoxia $\left(f_{i} \mathrm{O}_{2}=0.21\right)$ for $24 \mathrm{~h}$. We evaluated apoptosis, myelination, and oligodendrocyte maturation with immunohistochemistry, gene and protein analyses, and in vivo diffusion tensor magnetic resonance imaging (MRI). Consistent with previous studies, we found an increase in apoptosis of oligodendrocytes as determined by TUNEL+ staining in Olig2+ cells in white matter, cortex, thalamus, and hippocampus following exposure to hyperoxia with no additional effect of cell-free $\mathrm{Hb}$. A transient increase in the mRNA expression of intercellular adhe-
\end{abstract}

\begin{tabular}{ll}
\hline KARGER & ( ) 2020 The Author(s) Karger \\
& Published by S. Karger AG, Basel Open access \\
karger@karger.com & This article is licensed under the Creative Commons Attribution- \\
NonCommercial-NoDerivatives 4.0 International License (CC BY- \\
www.karger.com/dne \\
NC-ND) (http://www.karger.com/Services/OpenAccessLicense). \\
Usage and distribution for commercial purposes as well as any dis- \\
tribution of modified material requires written permission.
\end{tabular}

sion molecule 1 at $6 \mathrm{~h}$ was observed following combined exposure to cell-free $\mathrm{Hb}$ and hyperoxia. No indications of oligodendrocyte maturational delay or hypomyelination were observed after either insult, delivered separately or combined, as determined by immunohistochemistry, Western blot, and diffusion tensor MRI. In our model, exposure to circulatory cell-free $\mathrm{Hb}$, with or without concomitant hyperoxia, did not significantly alter brain white matter development.

(C) 2020 The Author(s)

Published by S. Karger AG, Basel

\section{Introduction}

Diffuse white matter injury is a neuroradiological finding frequently encountered in neonates with critical congenital heart defects $[1,2]$. An evolving understanding of the mechanisms of immature oligodendrocyte injury has elucidated that maturational arrest is, besides apoptosis, an important mechanism leading to myelination failure $[3,4]$.

In open heart surgery, cardiopulmonary bypass (CPB) circulation exposes the developing body to circulating 
cell-free hemoglobin $(\mathrm{Hb})$ and hyperoxemia [5-7]. Cellfree $\mathrm{Hb}$ and its metabolites are highly reactive molecules with oxidative and proinflammatory effects. Some of the damaging properties of cell-free $\mathrm{Hb}$ are likely due to extravasation $[8,9]$ and endothelial integrity disruption $[10$, 11]. Systemic inflammation in the neonatal period has been shown to initiate microglial activation and subsequent injury to the developing oligodendrocyte $[12,13]$. Hyperoxia is known to challenge the immature oligodendrocyte population, introducing apoptotic pathway activation, maturation failure, and ensuing hypomyelination [14-16]. Data from porcine models have demonstrated that oligodendrocyte maturation was arrested after exposure to CPB circulation $[17,18]$.

The present study employed a small animal model to delineate potential white matter developmental alterations in the immature brain after exposure to circulating cell-free $\mathrm{Hb}$ and/or hyperoxemia. We evaluated alterations with immunohistochemistry (IHC), protein- and mRNA analyses, and in vivo magnetic resonance imaging (MRI) in the recently described postnatal day 6 (P6) rat pup model of systemic cell-free $\mathrm{Hb}$ and hyperoxia exposure [19]. We hypothesized that circulating cell-free $\mathrm{Hb}$ and hyperoxia would, by activating inflammatory and oxidative pathways, pose a significant burden on developing oligodendrocytes and result in identifiable white matter alterations. In addition, we anticipated that this combined exposure would result in a worse outcome than exposure to a single insult.

\section{Materials and Methods}

\section{Animals}

The experiments were performed on 297 Wistar rat pups (Scanbur Research A/S Denmark) from 26 litters. All pups were nursed and fed by lactating dams throughout the study. Animals were handled according to the Swedish Animal Welfare legislation. The research protocol was approved by the Swedish Animal Ethics Committee in Lund, Sweden.

\section{Preparation of Human Cell-Free $\mathrm{Hb}$}

Human cell-free $\mathrm{Hb}$ was purified, as previously described [20], from human adult red blood cells obtained from the blood center in Lund, Sweden. The $\mathrm{Hb}$ concentration was quantified with a plasma/low Hb system (Hemocue ${ }^{\circledR}$, Sweden). Briefly, Hb was dissolved in Ringer's acetate (Baxter, USA) to a concentration of 200 $\mathrm{mg} / \mathrm{mL}$. To purify $\mathrm{Hb}$ from endotoxin contamination, we used the endotoxin-removing product, EndoTrap ${ }^{\circledR}$ (Hyglos GmbH, Germany), as described by the manufacturer. The absolute purity of $\mathrm{Hb}$ from contamination with endotoxin was determined with the QCL-1000 ${ }^{\mathrm{TM}}$ Endpoint Chromogenic LAL Assay (Lonza, Switzerland), as described by the manufacturer.

White Matter Development after

Exposure to Cell-Free $\mathrm{Hb}$ and Hyperoxia
Exposure to Circulating Cell-Free Human $\mathrm{Hb}$ and Hyperoxia

The experimental procedure has been thoroughly described previously [19]. Briefly, litter-mixed P6 Wistar rat pups were randomized to one of the following 4 treatment groups: $\mathrm{HH}$ : intraperitoneal cell-free $\mathrm{Hb}$ injections and exposure to hyperoxia; $\mathrm{HN}$ : intraperitoneal cell-free $\mathrm{Hb}$ injections and exposure to normoxia; $\mathrm{VH}$ : intraperitoneal vehicle injections and exposure to hyperoxia; and $\mathrm{VN}$ : intraperitoneal vehicle injections and exposure to normoxia. Rat pups were sedated with isoflurane in room air and injected intraperitoneally with a body-weight-based dose of cell-free $\mathrm{Hb}$ (purified as described above), or with the same volume of vehicle (Ringer's acetate, Baxter). We calculated the dose of cell-free $\mathrm{Hb}$ for intraperitoneal administration, as described by Jungner et al. [19], with the aim of achieving a plasma concentration of $\sim 3 \mathrm{mg} / \mathrm{mL}$.

After recovery, pups were placed in a neonatal incubator (Dräger $8000^{\circledR}$; Drägerwerk AG, Germany) together with a lactating dam for exposure to hyperoxia $\left(\mathrm{f}_{\mathrm{i}} \mathrm{O}_{2}: 0.8\right)$ or normoxia $\left(\mathrm{f}_{\mathrm{i}} \mathrm{O}_{2}\right.$ : $0.21)$ for $24 \mathrm{~h}$. Oxygen concentrations in the incubators were monitored with a built-in monitoring system and cross-checked with a portable oxygen monitor (Teledyne MX300-I; Teledyne Analytical Instruments, USA). The incubator temperature was maintained at a constant temperature of $28^{\circ} \mathrm{C}$. After the 24 -h exposure to hyperoxia or normoxia, animals were housed in room air and room temperature until termination. Animals allocated to IHC were perfused, as described below, at P7 ( $24 \mathrm{~h}$ after injection) and P11 (120 h after injection). Animals allocated to protein and gene analyses in brain tissue were terminated by decapitation at P6 (6 h after injection), P7 (24 h after injection), and P11 (120 h after injection). Brains were excised and snap-frozen on dry ice before analyses. Animals allocated to in vivo MRI analyses were terminated by decapitation immediately after the MRI examination at P7 (24 h after injection), P9 (72 h after injection), and P21 (360 $\mathrm{h}$ after injection). The overall study design is illustrated in Figure 1.

\section{Immunohistochemistry}

For IHC, pups were transcardially perfused at P7 and P11 with phosphate-buffered saline (PBS, $\mathrm{pH} 7.4$ ) followed by $4 \%$ paraformaldehyde (buffered with PBS, $\mathrm{pH}$ 7.4). After fixation, the brains were dissected from the skull, postfixed by immersing in $4 \%$ paraformaldehyde at $4{ }^{\circ} \mathrm{C}$, and embedded in paraffin. Prior to antibody staining, $10-\mu \mathrm{m}$ coronal sections $(-3.72 \pm 0.7 \mathrm{~mm}$ from the breg$\mathrm{ma}$ ) were rehydrated in $10 \mathrm{mM}$ sodium citrate buffer $(\mathrm{pH} 6.0,0.2 \%$ Triton X-100), and antigen retrieval was performed at $100^{\circ} \mathrm{C}$ (in a preheated water bath) for $30 \mathrm{~min}$. Sections were mounted on slides and blocked with $1 \%$ bovine serum albumin, $0.3 \%$ cold fish skin gelatin, and $0.1 \%$ Tween-20 dissolved in Tris-buffered saline. Next, the slides were incubated with primary antibodies (described in more detail below) overnight at $4{ }^{\circ} \mathrm{C}$, followed by appropriate secondary antibody incubation for $1 \mathrm{~h}$ at room temperature. Sections were counterstained with 4',6-diamidino-2-phenylindole (DAPI, $1 \mu \mathrm{g} / \mathrm{mL}$; Invitrogen, USA).

Myelination was evaluated at $\mathrm{P} 11$ with the primary mouse antirat myelin basic protein antibody (MBP, diluted 1:100, SMI-99; Sternberger Monoclonals, USA). Apoptotic degeneration of oligodendrocytes was evaluated at $\mathrm{P} 7$ by colabeling with polyclonal rabbit anti-Olig2 (diluted 1:100; Millipore, Germany) and terminal deoxynucleotidyl transferase-mediated biotinylated dUTP nick end labeling (TUNEL, in situ cell death detection kit, FITC; Roche, 
Fig. 1. Diagram of the experimental setup. The time points of blood/tissue sampling and in vivo MRI are indicated with arrows. $\mathrm{HH}$, intraperitoneal injection of cell-free $\mathrm{Hb}$ and exposure to hyperoxia; $\mathrm{HN}$, intraperitoneal injection of cell-free $\mathrm{Hb}$ and exposure to normoxia; $\mathrm{VH}$, intraperitoneal injection of vehicle and exposure to hyperoxia; $\mathrm{VN}$, intraperitoneal injection of vehicle and exposure to normoxia; $\mathrm{f}_{\mathrm{i}} \mathrm{O}_{2}$, fraction of inhaled oxygen; MRI, magnetic resonance imaging.

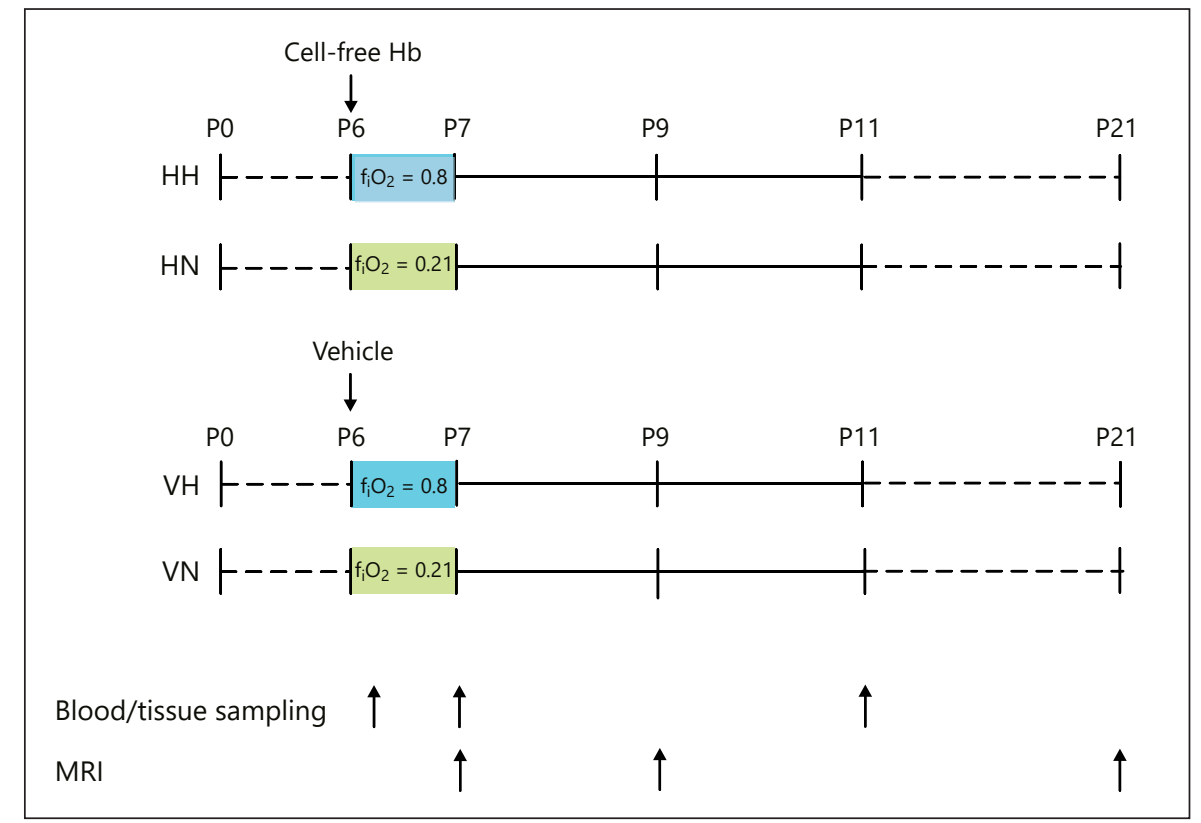

Germany), both performed according to the manufacturer's instructions. Mature oligodendrocytes were identified at P11 by staining with a monoclonal mouse antibody (clone CC1) that targeted adenomatous polyposis coli (anti-APC, referred to as CC1; diluted 1:100; Calbiochem, Germany). All primary antibodies were detected with appropriate secondary antibody staining (for rabbit: anti-rabbit Alexa Fluor 594, diluted 1:500; for mouse: antimouse Alexa Fluor 488, diluted 1:500; both Invitrogen).

To evaluate cellular, and particularly oligodendrocyte, apoptotic degeneration, we analyzed TUNEL/Olig2-stained brain sections with confocal microscopy (A1plus, Eclipse Ti, with NIS Elements AR software; Nikon, Germany) and a $\times 20$ objective. Images were acquired with 2 laser lines (laser diode, $405 \mathrm{~nm}$; $\mathrm{Ar}$ laser, $514 \mathrm{~nm}$; G-HeNe laser, $543 \mathrm{~nm}$ ) and 3 different filters (450/50-405 LP, 515/20-540 LP, and 585/65-640 LP). Confocal $z$-stacks of 10 - $\mu \mathrm{m}$ thickness ( $z$-plane distance, $1 \mu \mathrm{m}$ ) were converted into 2 -dimensional images with maximum intensity projections (performed with NIS Elements AR software). TUNEL/ Olig2 double-positive cells were counted in 16 defined, nonoverlapping regions of interest (ROIs, each ROI: 396,900 $\mu^{2}$; ROIs included: $3 \times$ cortex; $3 \times$ hippocampus; $3 \times$ thalamus; and $7 \times$ white matter, which included $1 \times$ corpus callosum, $1 \times$ cingulate, and $1 \times$ deep cortical white matter; $1 \times$ internal capsule; and $3 \times$ external capsule). Four hemispheres per animal were analyzed. For MBP/Olig2 and CC1/Olig2 co-stained sections, we visualized the defined ROIs with fluorescence microscopy $(\times 20$ objective; Axioplan; Zeiss, Germany) and acquired images with a CCD camera (Microfire; AVT Horn, Germany). We analyzed 7 ROIs (each: $189,135 \mu \mathrm{m}^{2}$; $3 \times$ thalamus; $3 \times$ white matter, including deep cortical white matter, internal and external capsules; and $1 \times$ cingulum) in 2 hemispheres per animal. MBP expression was quantified as the fraction of positive area after converting images into binary images with ImageJ software. Olig2- and CC1-positive cells were counted manually after auto-contrast and background subtraction.

\section{SDS-PAGE and Western Blotting}

Protein analyses of MBP with SDS-PAGE and Western blotting were conducted after animals were terminated by decapitation at P11. The brains were removed from the skull, separated into left and right hemispheres, snap-frozen on dry ice immediately after excision, and stored at $-80^{\circ} \mathrm{C}$ until further use. Total protein was extracted from brain tissues by homogenizing $150 \mathrm{mg}$ of frozen brain tissue on ice in cell extraction buffer (Invitrogen, cat. No. FNN0011), supplemented with complete mini-EDTA-free proteinase inhibitor cocktail tablets, reconstituted according to manufacturer instructions (Roche, cat. No. 11836170001). Protein concentrations were determined with the Pierce ${ }^{\mathrm{TM}} \mathrm{BCA}$ protein assay kit (Thermo Scientific, USA).

SDS-PAGE was run with standard protocols. Briefly, 50- $\mu \mathrm{g}$ protein samples were separated on stain-free, 4-20\% gels (Bio-Rad Laboratories, USA) at $200 \mathrm{~V}$ for 30-40 min under reducing conditions with a molecular weight (MW) standard (Precision Protein Plus All Blue Marker; Bio-Rad). The separated proteins were transferred to polyvinylidene difluoride membranes (PVDF; Bio-Rad) and blocked with $5 \%$ blocker-grade dry milk (Bio-Rad). The membranes were then incubated with antibodies against MBP (diluted $1: 1,000, \mathrm{MBP}$ [D8X4Q] $\mathrm{XP}^{\circledR}$ rabbit mAb; Cell Signaling, USA) and $\beta$-actin (diluted 1:10,000, cAnti- $\beta$-actin antibody [AC-15] ab6276; monoclonal mouse anti-human $\beta$-actin; Abcam, UK). Western blotting was performed with the following secondary antibodies: swine anti-rabbit IgG conjugated to horseradish peroxidase (HRP) or goat anti-mouse IgG conjugated to HRP (diluted 1:5,000 and 1:1,500, respectively; Dako A/S, Denmark). The bands were detected in a ChemiDoc ${ }^{\mathrm{TM}}$ XRS unit (Bio-Rad, USA). We quantified the relative concentrations of MBP (bands 1 and 2, MW 12-18 $\mathrm{kDa}$ ), Olig2 (MW $\sim 37 \mathrm{kDa})$, and $\beta$-actin (MW $\sim 42 \mathrm{kDa}$ ) with densitometry in Image Lab software (Bio-Rad). MBP levels were normalized to endogenous $\beta$-actin levels. 
mRNA Analyses

For mRNA analyses, animals were terminated by decapitation at P6, P7, and P11. The brains were removed from the skull, separated into left and right hemispheres, snap-frozen on dry ice immediately after excision, and stored at $-80{ }^{\circ} \mathrm{C}$ until further use. Total RNA was extracted from $30 \mathrm{mg}$ of brain tissue homogenate (1 hemisphere) with the RNeasy ${ }^{\circledR}$ mini kit (Qiagen, USA). The optical density ratios $(260 / 280 \mathrm{~nm})$ of RNA samples were always above 1.95. Reverse transcription of $1.0 \mu \mathrm{g}$ pooled total RNA was performed according to the manufacturer instructions. Pools were formed by combining equal amounts of genetic material from 8 individual animals into 1 sample. Each pool was representative of a single experimental group at a specific time point. We performed reverse transcription with the $\mathrm{RT}^{2}$ first strand kit (Qiagen). We performed quantitative polymerase chain reaction (qPCR) arrays for 4 different signaling pathways (oxidative stress, apoptosis, NFkB signaling, and tight junctions, all from Qiagen). Based on the results from the qPCR arrays, we performed confirmatory quantitative analyses, with qPCR primer assays (Qiagen) for tumor necrosis factor (TNF), interleukin (IL)-10, claudin 2 (Cldn2), glutathione peroxidase 6 (GPX6), superoxide dismutase 3 (SOD3), IL$1 \alpha$, IL-1 $\beta$, heme oxygenase 1 (HMOX-1), and intercellular adhesion molecule 1 (ICAM-1). The qPCR array and primer assays were performed according to the manufacturer's instructions (Qiagen).

We analyzed gene expression with the $\mathrm{RT}^{2}$ SYBR Green qPCR Master Mix (Qiagen). We performed amplification at appropriate temperatures for 40 cycles in a CFX Connect Thermal Cycler ${ }^{\mathrm{TM}}$ (Bio-Rad). The data were analyzed with Bio-Rad CFX Maestro 1.1 software (Bio-Rad). Data were normalized to the expression of ribosomal protein lateral stalk subunit P1 with the standardized quantitative method for normalization. The fold change values were calculated by normalizing to the $\mathrm{VN}$ (control) group values at the corresponding time points.

\section{In vivo MRI Data Acquisition}

In vivo brain MRI was performed at P7, P9, and P21. Anesthesia was induced with $4 \%$ isoflurane. Animals were placed in a custom-made holder. We monitored respiration and temperature with a pressure-sensitive pad (SA Instruments, Inc., Stony Brook, NY, USA) and a thermal probe (SA Instruments, Inc.) placed under the pup's abdomen. Anesthesia was maintained with isoflurane in $\mathrm{O}_{2}$, and the isoflurane concentration was adjusted to maintain respiratory rate between 60 and 80 breaths/min. To prevent hypothermia, we increased the ambient temperature with a heating module fan (SA Instruments, Inc.).

MRI scans were performed on a 9.4-T horizontal bore animal scanner (Agilent Inc., USA), equipped with a 115/60 HD (High Duty Cycle) gradient coil with a $6-\mathrm{cm}$ bore $(1,000 \mathrm{mT} / \mathrm{m}$ strength). For transmitting and receiving signals, we used the milli40 (Agilent Inc.), a millipede volume coil with quadrature detection and a 40$\mathrm{mm}$ inner diameter. Diffusion tensor imaging was performed with a standard bipolar pulse field gradient diffusion sequence with multi-shot echo planar imaging readout. We applied the following parameters: $\mathrm{TE}=13.21 \mathrm{~ms}$, $\mathrm{TR}=4,000 \mathrm{~ms}$, matrix $=96^{2}$, spectral width $=250 \mathrm{kHz}$, field of view $=20 \times 20 \mathrm{~mm}$, slice thickness $=0.60$ $\mathrm{mm}$ with no gap between slices, and the total scan time was $10 \mathrm{~min}$.

The mean diffusivity and fractional anisotropy (FA) were calculated with a conventional diffusion-weighted imaging analysis [21]. The postprocessing and analysis software were written in MATLAB $^{\circledR}$ (The MathWorks, Natick, MA, USA) [22]. Data for T2

White Matter Development after

Exposure to Cell-Free $\mathrm{Hb}$ and Hyperoxia maps were acquired with the same positioning as described above with the multi-echo multi-slice sequence, a multi-spin echo acquisition given by $\mathrm{TR}=3.502 \mathrm{~ms}$, echo times $\mathrm{TE}=6.104 \mathrm{~ms}, \mathrm{~N}_{\mathrm{TE}}=$ 16 , echo spacing $=6.104 \mathrm{~ms}$, and a total acquisition time of $7 \mathrm{~min}$ $28 \mathrm{~s}$. T2 maps were derived on a pixel-by-pixel basis with nonlinear least-square curve fitting based on an exponential decay equation:

$S=S_{0} \times \exp \left(-\frac{T E}{T 2}\right)$,

where $\mathrm{S}_{0}$ is the pixel intensity at $\mathrm{TE}=0 \mathrm{~ms}$. Data quantification was performed in ImageJ analysis software (https://imagej.nih.gov/ij/) with an in-house plugin. For further technical specifications, please refer to the supplementary material.

FA values were selected as the primary means of evaluating white matter integrity. During the analyses, the examiner was blinded to the study group but not to the postnatal age. FA values were analyzed in 5 non-overlapping ROIs in the corpus callosum, internal capsules (bilateral), and trigeminal nerves (bilateral). Each anatomic location was analyzed on 2 (internal capsule and trigeminal nerve) or 3 (corpus callosum) consecutive slices, selected relative to anatomic landmarks visualized on T2-weighted images. We selected ROIs representing different maturational stages. Trigeminal nerve myelination starts at P7, and myelination of the corpus callosum and the internal capsule start at P14 and P12, respectively. Quality was assessed by confirming an increase in FA values with increasing gestational age, and by confirming that FA values differed with divergent maturational timelines at separate anatomical locations at the same gestational age.

\section{Statistics}

All statistics were performed with GraphPad Prism8 software (La Jolla, CA, USA). Results are presented as medians and interquartile ranges, unless otherwise stated. Comparisons between groups were performed assuming parametric data distributions, with analysis of variance (ANOVA) and Dunnett's test for multiple group comparisons. When performing multiple group comparisons, all groups were compared to the VN group. Values of $p<0.05$ were considered significant.

\section{Results}

\section{Hyperoxia, but Not Cell-Free Hb Exposure, Increased Oligodendrocyte Apoptosis}

To examine the impact of circulating cell-free $\mathrm{Hb}$ and hyperoxia as single and combined insults on the developing white matter, we first evaluated oligodendrocyte apoptosis. We analyzed oligodendrocytes by double staining with TUNEL, a marker of apoptosis, and Olig2, a marker of oligodendrocytes in all maturational stages.

At P7, IHC analysis revealed a significant increase in the number of apoptotic oligodendrocytes (TUNEL/ Olig2 double-positive cells; Fig. 2) in the $\mathrm{VH}$ and $\mathrm{HH}$ groups compared to the VN group. This increase was significant in white matter (VH: $p=0.0001 ; \mathrm{HH}: p=$ $0.002)$, cortex (VH: $p=0.0003 ; \mathrm{HH}: p=0.004)$, thalamus 


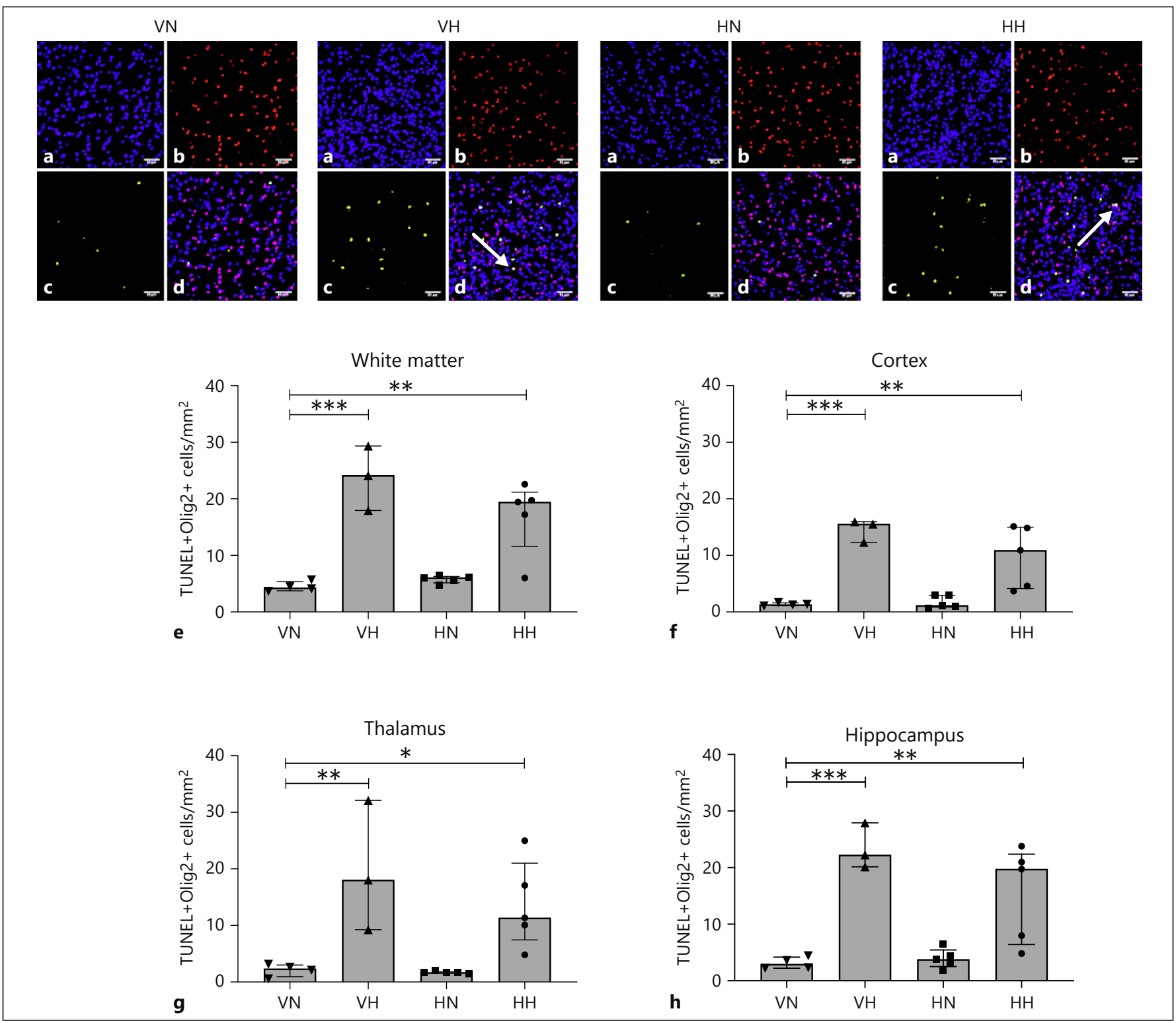

Fig. 2. TUNEL/Olig2 staining of rat pup brain sections after exposure to hyperoxia $(\mathrm{VH})$, circulating cell-free $\mathrm{Hb}(\mathrm{HN})$, both insults $(\mathrm{HH})$, or neither insult (VN). Representative images were acquired at P7 (24 h after injection) in all experimental groups. Scale bar, 50 $\mu \mathrm{m}$. a DAPI staining of cell nuclei (blue). b Olig2+ cells (red). c TUNEL+ cells (green). d Merged image. White arrows indicate co-labeled cells. TUNEL+/Olig2+ cells were quantified in white

(VH: $p=0.007 ; \mathrm{HH}: p=0.041$ ), and hippocampus (VH: $p=0.0005$; HH: $p=0.006$ ). Notably, the double HH exposure did not have any additional effect on the number of TUNEL/Olig2 double-positive cells in any of the brain regions analyzed compared to the hyperoxia exposure alone ( $\mathrm{VH})$. Moreover, rat pups in the HN group did not matter (e), cortex (f), thalamus (g), and hippocampus (h) at P7. Results are presented as medians and interquartile ranges; $n=3-5$. ${ }^{*} p<0.05,{ }^{* *} p<0.01,{ }^{* * *} p<0.001$. DAPI, $4^{\prime}, 6$-diamidino-2phenylindole; TUNEL, terminal deoxynucleotidyl transferase dUTP nick end labeling; P7, postnatal day 7; Olig2, oligodendrocyte transcription factor 2 . 

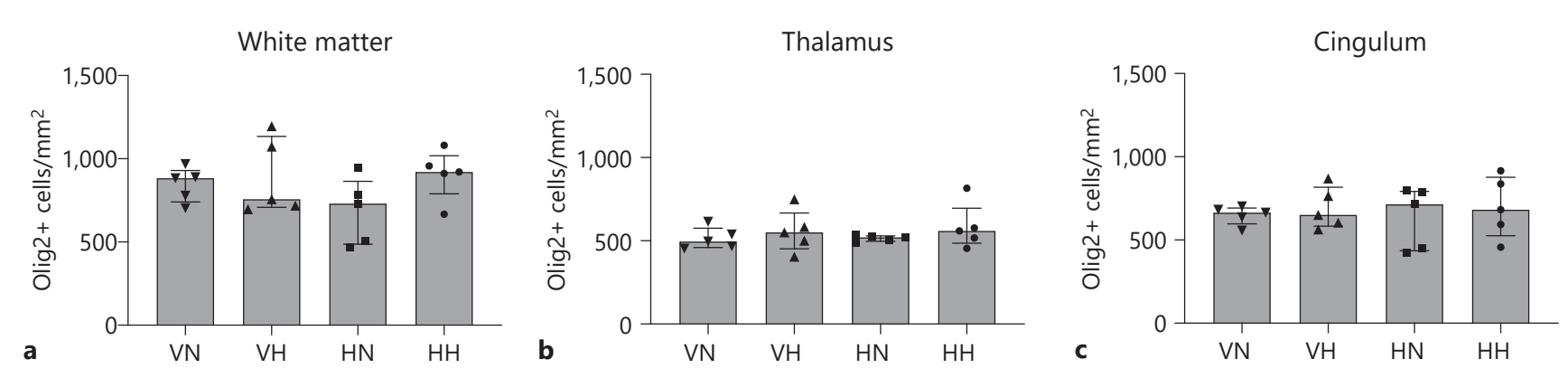

Fig. 3. Quantification of the total number of Olig2+ cells in different parts of the rat brain in groups exposed to hyperoxia (VH), circulating cell-free $\mathrm{Hb}(\mathrm{HN})$, both insults $(\mathrm{HH})$, or neither insult (VN). Rats were sacrificed on postnatal day 11 (120 h after injection), and Olig2+ staining was evaluated in white matter (a), thalamus (b), and cingulum (c). Results are presented as medians and interquartile ranges; $n=5$ (a-c). Olig2, oligodendrocyte transcription factor 2 .

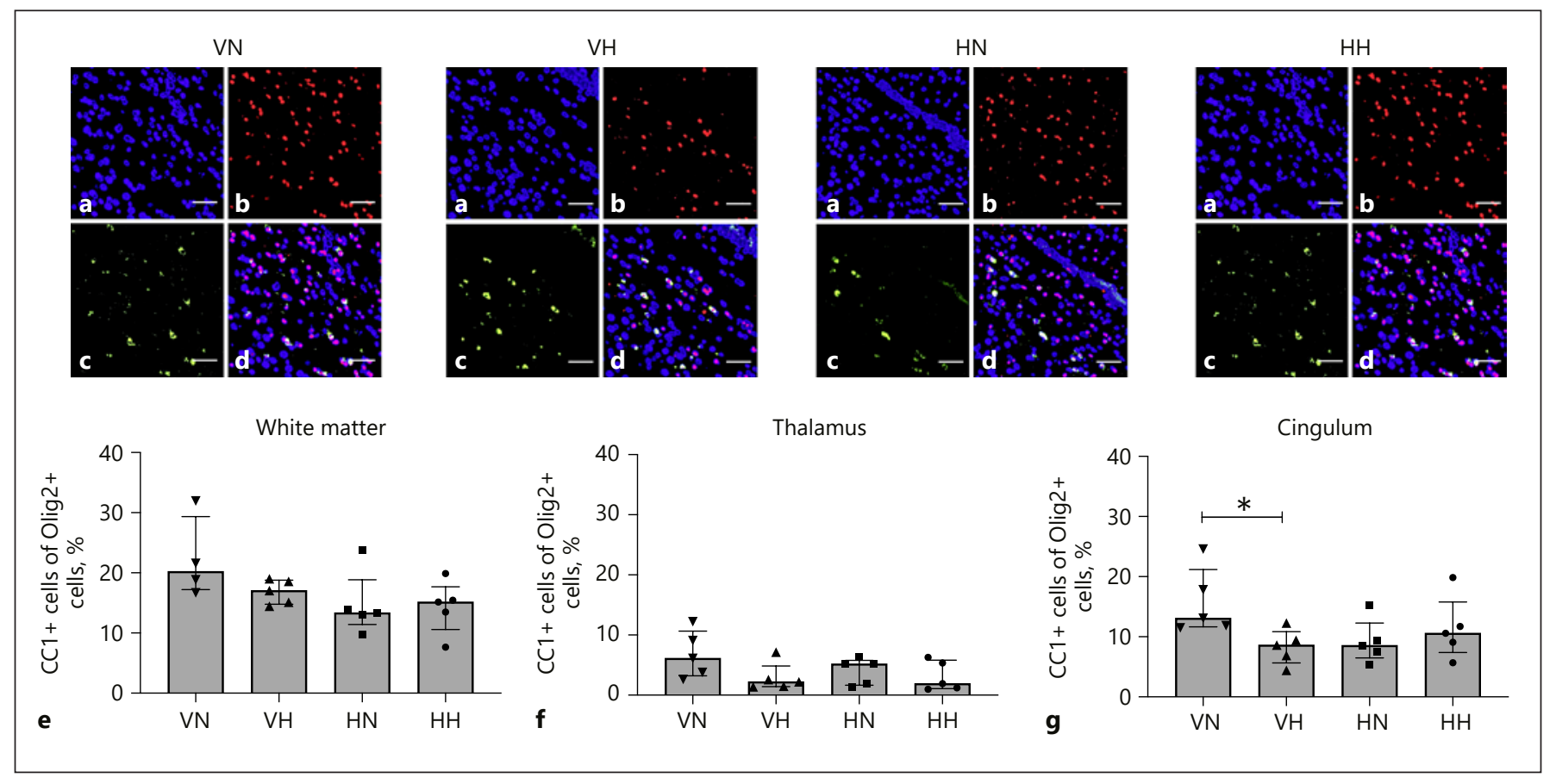

Fig. 4. Immunohistochemistry analysis of oligodendrocyte transcription factor (Olig)2+/adenomatous polyposis coli clone $(\mathrm{CC} 1+)$ cells in rat pup brain sections after exposure to hyperoxia $(\mathrm{VH})$, circulating cell-free $\mathrm{Hb}(\mathrm{HN})$, both insults $(\mathrm{HH})$, or neither insult (VN). Representative images were acquired on postnatal day 11 (P11) (120 h after injection) in all experimental groups. Scale

Despite our observation that apoptosis increased in oligodendrocytes at P7 (Fig. 2), we found no significant difference in the total number of Olig2-positive cells analyzed at P11 with IHC in the different brain ROIs (white matter, thalamus, and cingulum; Fig. $3 \mathrm{a}-\mathrm{c}$ ).

White Matter Development after

Exposure to Cell-Free $\mathrm{Hb}$ and Hyperoxia bar, $50 \mu \mathrm{m}$. a DAPI staining of cell nuclei (blue). b Olig2+ cells (red). c CC1+ cells (green). $\mathbf{d}$ Merged image. e-g Quantification of the percentage of CC1+ cells among Olig2 + cells in white matter (e), thalamus (f), and cingulum (g) on P11. Results are presented as medians and interquartile ranges; $n=5$. ${ }^{*} p<0.05$. DAPI, 4',6-diamidino-2-phenylindole.

\section{Cell-Free Hb and Hyperoxia Do Not Affect Maturation of Immature Oligodendrocytes}

We evaluated whether circulating cell-free $\mathrm{Hb}$ and/or hyperoxia affected oligodendrocyte maturation by analyzing $\mathrm{CC} 1$, a protein expressed on the cell bodies of ma-

Dev Neurosci 2019;41:234-246 
Fig. 5. MRI analysis of white matter microstructural organization in rat pup brain after exposure to hyperoxia ( $\mathrm{VH})$, circulating cell-free $\mathrm{Hb}(\mathrm{HN})$, both insults $(\mathrm{HH})$, or neither insult (VN). Representative MRI images show a rat brain on postnatal day 7 (P7). a Diffusion tensor imaging with the capsula interna dexter (c int dx) marked with a white line. b Corresponding T2weighted image in the same animal. c Fractional anisotropy (FA) measured in the $\mathrm{c}$ int $\mathrm{dx}$ in all experimental groups at all time points. d T2 relaxation times measured in the $\mathrm{c}$ int $\mathrm{dx}$ in all experimental groups at all time points. c, d Median values (bars) and individual animals (symbols) are displayed. $n=3-4$ for each group.
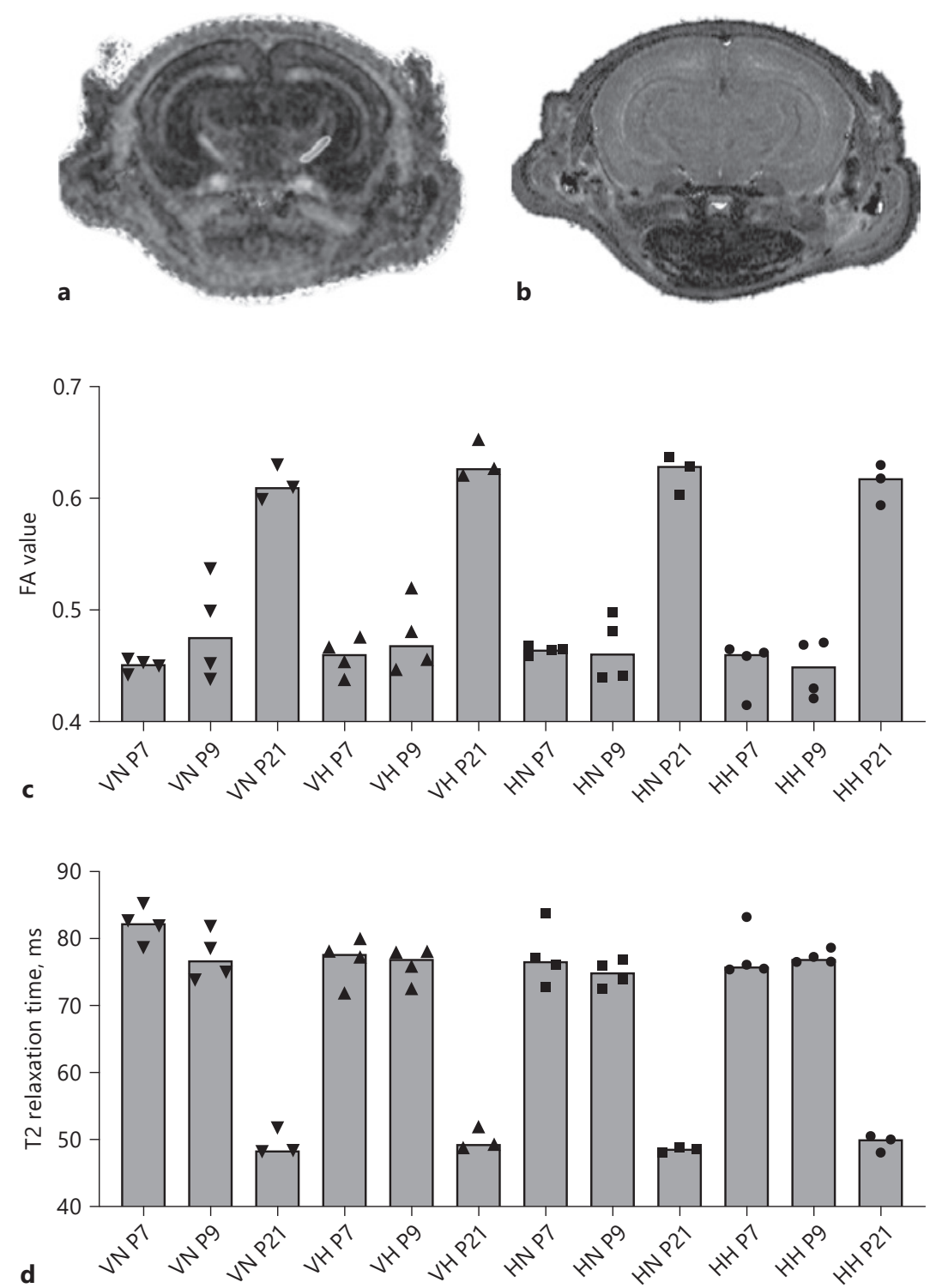

ture oligodendrocytes at P11. When we double-labeled $\mathrm{CC} 1$ and Olig2, we observed a trend towards reducing the percentage of CC1-positive cells within the Olig2-positive population. This reduction was significant in the cingulum region $(p=0.047)$ in animals in the $\mathrm{VH}$ group compared to those in the VN group (Fig. $4 \mathrm{a}-\mathrm{c}$ ).

To evaluate the effects of circulating cell-free $\mathrm{Hb}$ and/or hyperoxia on oligodendrocyte maturation, we calculated FA values and T2 relaxation times based on the MRI exam at P7, P9, and P21. In the developing brain of an unexposed animal, we expected an increase in FA values with increasing postnatal age. This increase occurs when the white matter microstructural organization becomes more linear and, thus, more anisotropic. Additionally, we expected a reduction in the $\mathrm{T} 2$ relaxation time reflecting reduced tissue water content, which occurs in increased myelination. However, we found no differences in either the FA values or the $\mathrm{T} 2$ relaxation times between experimental groups at any of the time points in the evaluated ROIs (Fig. 5a-d).

\section{Cell-Free Hb and Hyperoxia, Alone or Combined,}

Do Not Reduce Myelination

To assess whether cell-free $\mathrm{Hb}$ exposure as a single insult or in combination with hyperoxia had an impact on 


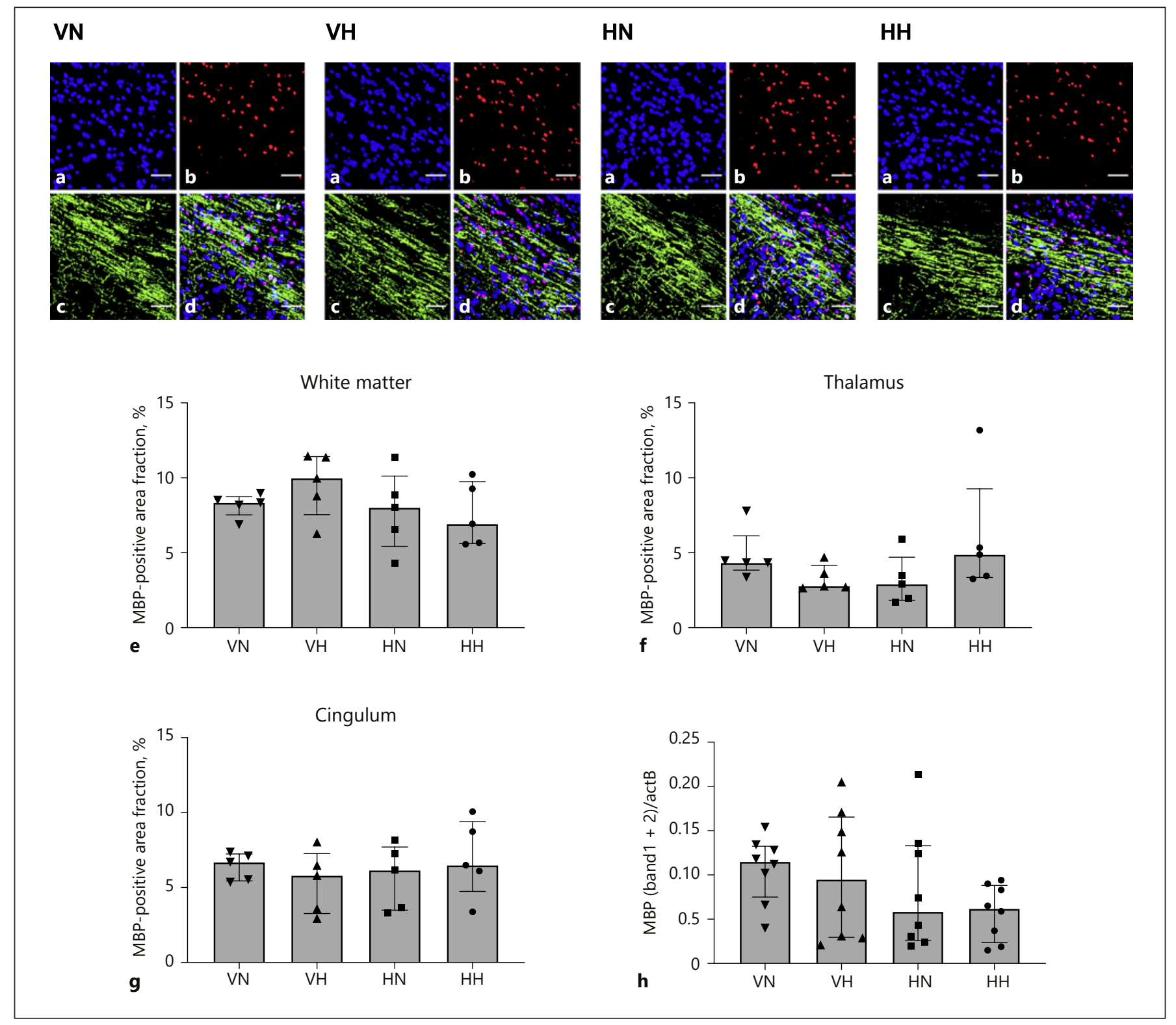

Fig. 6. Immunohistochemistry analysis of myelin formation in rat pup brain after exposure to hyperoxia $(\mathrm{VH})$, circulating cell-free $\mathrm{Hb}(\mathrm{HN})$, both insults $(\mathrm{HH})$, or neither insult (VN). a-d Representative images show myelin basic protein (MBP) in brain sections on postnatal day 11 (P11) (120 h after injection) in all experimental groups. Scale bar, $50 \mu \mathrm{m}$. a DAPI staining of cell nuclei (blue). b Olig2+ cells (red). c MBP (green). d Merged image. e-g Quanti-

the formation of myelin, we examined the degree of myelination by staining MBP in IHC analyses. At P11, we found no significant differences in myelination in any of the 3 regions examined (Fig. 6a-c). This result was confirmed by Western blot analysis of the MBP content in total brain homogenate (one hemisphere) at P11 (Fig. 6h). fication of MBP protein content displayed as a fraction of the region of interest area (\%) in white matter (e), thalamus (f), and cingulum (g). $\mathbf{h}$ Western blot analysis of MBP content $(\mathrm{MBP}$ band $1+2)$ compared to endogenous $\beta$-actin (actB) in brain homogenates on P11. Results are presented as medians and interquartile ranges; $n=5$ (a-c) and $n=8$ (d). DAPI, 4',6-diamidino-2-phenylindole; Olig2, oligodendrocyte transcription factor 2 .

\section{Circulating $\mathrm{Hb}$ and Hyperoxia Induce a Transient \\ Increase in ICAM-1 Expression}

To understand whether cell-free $\mathrm{Hb}$ and/or hyperoxia induced activation of any major signaling pathways in the neonatal rat brain, we performed qPCR array analyses on pooled material from the total brain homogenates of the respective 


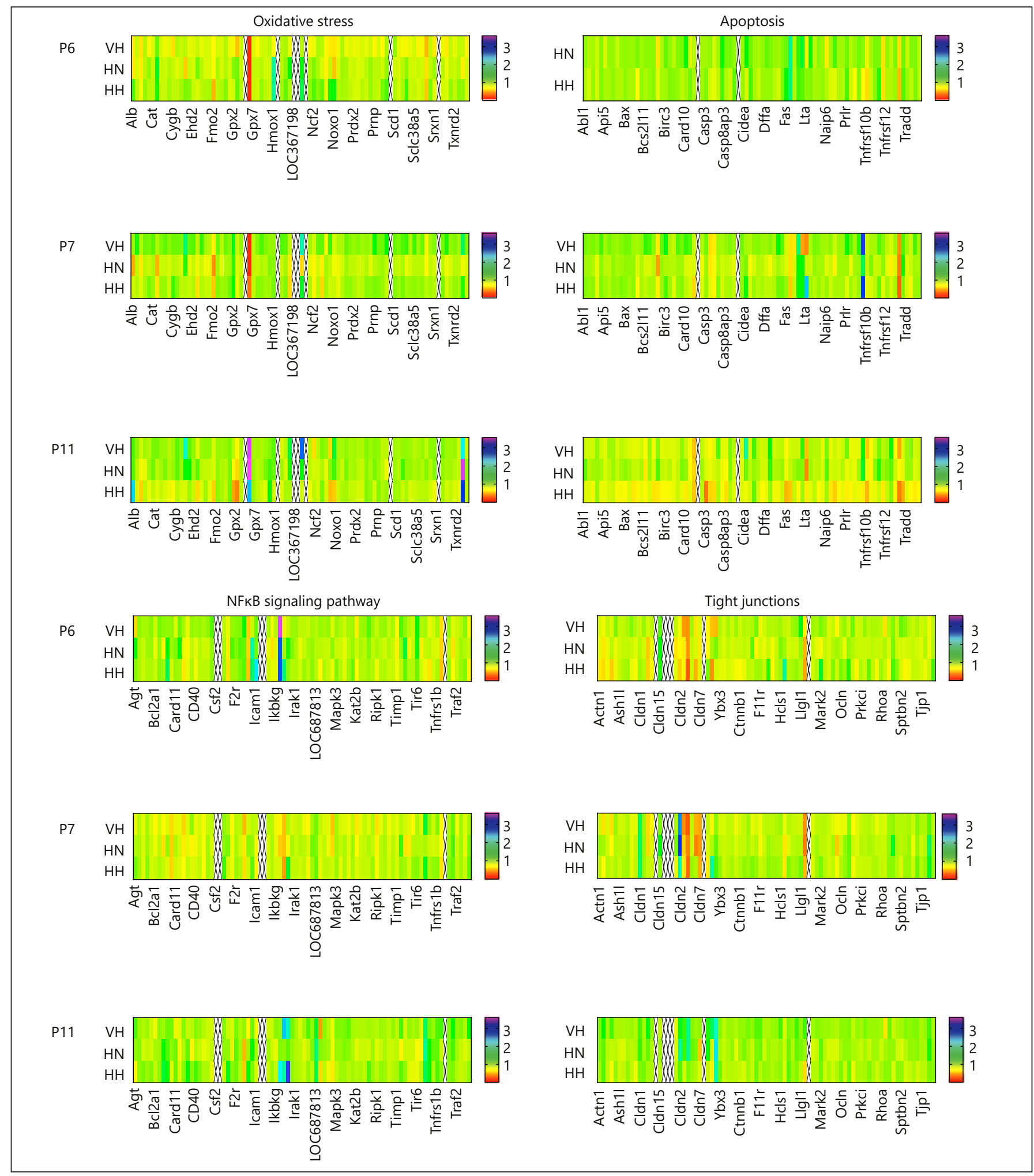

Fig. 7. qPCR array analysis of 4 different pathways of injury in pooled rat pup brain tissues after exposure to hyperoxia $(\mathrm{VH})$, circulating cell-free $\mathrm{Hb}(\mathrm{HN})$, or both insults $(\mathrm{HH})$. Oxidative stress, apoptosis, NFkB signaling pathway, and tight junction arrays were analyzed on postnatal day (P) 6 (6 h after injection), P7 (24 h after injection), and P11 (120 h after injection). Statistical analyses of intergroup comparisons were not performed as these analyses were performed on pooled material. 


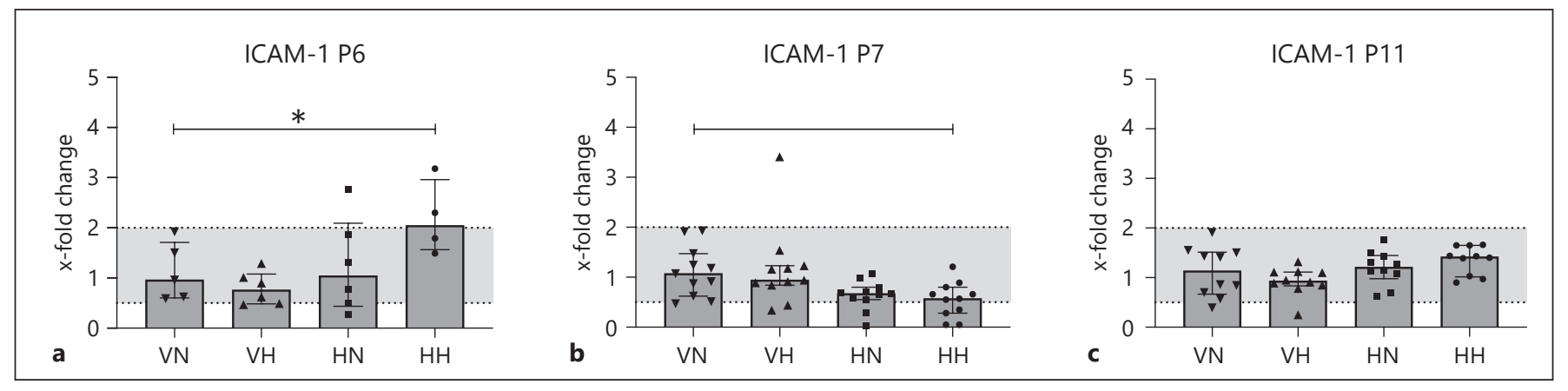

Fig. 8. qPCR analyses of ICAM-1 mRNA expression in individual rat pup brains after exposure to hyperoxia $(\mathrm{VH})$, circulating cellfree $\mathrm{Hb}(\mathrm{HN})$, both insults $(\mathrm{HH})$, or neither insult $(\mathrm{VN})$. Relative expression levels were evaluated on postnatal day (P) 6 (6 h after injection) (a), P7 (24 h after injection) (b), and P11 (120 h after injection) (c). Fold changes are displayed as medians and interquartile ranges; $n=4-11$. ${ }^{*} p<0.05$. ICAM-1, intercellular adhesion molecule 1 . groups at P6 (6 h after injection), P7 (24 h after injection), and P11 (120 h after injection). Pooled material was obtained by combining equal amounts of genetic material from 8 individual animals into 1 sample. These pooled samples were representative of each experimental group at each specific time point. We evaluated signaling pathways related to apoptosis, oxidative stress, tight junctions, and NFKB.

Overall, exposure to cell-free $\mathrm{Hb}$ and hyperoxia triggered subtle changes in brain-parenchymal gene expression, as shown in heat maps (Fig. 7). Among the pathways examined, the qPCR array analyses suggested group differences in the expression of endothelial adhesion molecules (ICAM-1), blood-brain barrier integrity (Cldn2), oxidative stress responses (GPX6, SOD3, and HMOX-1), and inflammatory responses (TNF, IL-10, IL-1 $\alpha$, and IL-1 $\beta$ ). Next, we analyzed these targets in qPCR analyses with gene material from individual animals. We observed a significant increase in ICAM-1 expression in the HH group at P6 ( $p=0.044)$ compared to the VN group. This group also displayed a significant reduction in ICAM-1 expression at P7 ( $p=0.049$; Fig. 8). The other selected genes showed no changes in expression in any group at any time point.

\section{Discussion}

In this study, we explored the impact of cell-free $\mathrm{Hb}$ and hyperoxia, separately and in combination, on white matter brain development in neonatal rats. We reaffirmed the previously described correlation between hyperoxia exposure and apoptosis induction. However, we found no marked effects on white matter brain development. The most probable explanation for our negative findings was that the insults were too subtle to cause im- pairments that could be detected with our analytical approach in our relatively small group sizes. Nevertheless, there are a number of important points to consider.

Exposure to circulating cell-free $\mathrm{Hb}$ alone did not cause any detectable white matter alterations. To explore this negative finding, we attempted to determine whether cell-free $\mathrm{Hb}$ could transfer across the blood-brain barrier. We performed an IHC analysis to detect cell-free $\mathrm{Hb}$ within the brain but failed to detect any quantitative differences in $\mathrm{Hb}$ labeling between the experimental groups in the corpus callosum, the anterior commissure, the lateral ventricles, or the choroid plexus (data not shown). It was previously proposed that the toxicity of cell-free $\mathrm{Hb}$ may increase when accompanied by an additional insult [23]. In our setting, the additional insult was hyperoxia. When considering a "double-hit" approach, it is important to take the concepts of sensitization and protection into account. Our primary hypothesis was that a hyperoxic environment would augment the oxidation of cellfree $\mathrm{Hb}$ and increase the production of reactive oxygen species and oxidized $\mathrm{Hb}$ metabolites, and thereby enhance the induction of inflammation. Previous clinical and experimental research has implied that inflammation may have a sensitizing effect on subsequent brain injuries in full-term newborn infants [24]; in contrast, experimental studies showed that an inflammatory insult prior to induced hyperoxia had a protective effect [16]. We hypothesized that the temporal relationship between both insults in this study (i.e., hyperoxemia and circulating cell-free $\mathrm{Hb}$ ) was fairly representative of the temporal relationship that occurs during CPB circulation in a clinical setting. However, in contrast to our hypothesis, we failed to find any augmented effect on brain injury with exposure to the combined insults. 
Our results contradicted previous reports that showed that hypomyelination occurred after hyperoxic exposure [15]. This discrepancy might be due to differences in the applied methodology between studies. For evaluating MBP, we employed an automated measurement of the area; in contrast, previous studies [15] utilized a semiquantitative scoring system. The automated measurements were later shown to result in smaller effect sizes [25]. Additionally, we studied litter-mixed experimental groups in order to avoid genetic traits in littermates that might confound the results. Litter-to-litter heterogeneity can be extensive [26]; thus, our approach of litter-mixing was more prone to variability. We did not control for litter effects in our statistical analyses. Finally, we acknowledge the fact that the relatively low number of rat pups in some of the experimental groups increased the risk of $\beta$-errors. In our opinion, the different group distributions were better depicted by our figures than by the (lack of) significant $p$ values.

Attempts to amplify the insult by increasing the plasma concentrations of cell-free $\mathrm{Hb}$ were not successful due to technical challenges. Increasing or prolonging the exposure to hyperoxia did not appear relevant, but an alternate approach to enhance the insults might be to amplify the effects of the hyperoxic insult by introducing a prehypoxic phase. Indeed, there are reports [27] on increased apoptosis in the entire oligodendrocyte population in an experimental setting that mimicked conditions during $\mathrm{CPB}$ when a prehypoxic phase was applied. That setting would have the additional advantage of bringing the experimental model closer to the clinical context, as a significant proportion of neonates with congenital heart defects are hypoxemic prior to open heart surgery.

This study had several limitations. Most of our data showed substantial variability within the experimental groups. The hyperoxic insult was easy to standardize, but the exposure to circulating cell-free $\mathrm{Hb}$ after an intraperitoneal injection was difficult to predict. As previously shown [19], considerable variation in circulating plasma cell-free $\mathrm{Hb}$ concentrations was observed, even after optimizing the intraperitoneal $\mathrm{Hb}$ administration conditions. In our experimental setting, we could not control the maximum levels of circulating cell-free $\mathrm{Hb}$, as this would have limited the experimental time frame to $3 \mathrm{~h}$ after injection. Moreover, we did not control for the sex of the pups, a factor of emerging importance, as recent research in rodent models has suggested that the response to noxious stimuli was gender specific $[28,29]$. Hence, we could not rule out sex as an explanation for the observed variability within the groups. There were no differences in weight between the groups at P6, nor were there any differences in weight gain between the experimental groups throughout the study (data not shown).

Most studies, including this study, that investigated white matter brain injuries focused on instantaneous effects after the imposed insults. However, as recently discussed in an extensive review [30], a long-term perspective is essential for a thorough understanding of the true consequences of perinatal brain damage. Subtle immediate effects in the perinatal period may result in adverse neurobehavioral outcomes in adolescence [31]. Therefore, another limitation of the present study was that we did not include any follow-up beyond P21, and we did not conduct neurobehavioral testing.

A particular limitation, which was important in the context of our study, was that, in contrast to humans, rats have the ability to produce endogenous ascorbic acid. Ascorbic acid is a powerful antioxidant in the setting of cell-free $\mathrm{Hb}$ toxicity [32]. This disadvantage must be accepted when investigating neonatal neuroprotection in relation to $\mathrm{Hb}$ toxicity in rodents. The only rodent incapable of endogenous ascorbic acid production is the guinea pig; however, in those animals, brain maturity at birth is far more accelerated [33].

Another potential limitation of our study was the use of sedation. All rat pups were sedated with isoflurane during the intraperitoneal injection of cell-free $\mathrm{Hb}$, during the MRI scans, and immediately prior to decapitation. We are well aware of the fact that volatile anesthetics are neurotoxic in rodents [34]; however, with sedation, the quality and reproducibility of the intraperitoneal injection increased substantially. We deemed this a solid argument for continuing our sedation regime. Moreover, exposure to volatile anesthetics, except during the MRI scans, was limited to a few minutes and did not differ between experimental groups.

In conclusion, this study explored the impact of cellfree $\mathrm{Hb}$ and hyperoxia exposure on brain white matter development in the neonatal rat. In our experimental setting, we could not show that circulating cell-free $\mathrm{Hb}$ augmented the damaging effect of induced hyperoxia on white matter brain development.

\section{Acknowledgments}

The authors wish to thank Karin Sjölund, Lund University, for excellent technical assistance, and Bo Holmqvist, ImaGene-iT Sweden, for involvement in the IHC analyses of cell-free $\mathrm{Hb}$ extravasation. 


\section{Statement of Ethics}

Animals were handled according to the Swedish animal welfare legislation. The research protocol was approved by the Swedish Animal Ethics Committee in Lund, Sweden.

\section{Disclosure Statement}

The authors declare no conflicts of interest.

\section{Funding Sources}

This study was funded by the Swedish Research Council (201702112), the Swedish Heart-Lung Foundation, ALF Government grants to Lund University, the Skåne Council Foundation for Research, Queen Silvia's Foundation, Swedish Foundation for Strategic Research (RBP14-0055) and Lilla Barnets Fond. None of the funding sources had any role in preparing the data or the manuscript.

\section{Author Contributions}

Åsa Jungner: conceptualization and design of the study; acquisition and interpretation of data; drafting the initial manuscript, and revising the manuscript; Suvi Vallius Kvist, Matteo Bruschettini, and Olga Romantsik: acquisition and analysis of data; revision of the manuscript; Claes Ekström, Adnan Bibic, and Rene In'T Zandt: acquisition, analysis, and interpretation of data; revision of the manuscript; Ivo Bendix, Josephine Herz, and Ursula Felderhoff-Mueser: analysis and interpretation of data; revision of the manuscript; Magnus Gram: conceptualization and design of the study; acquisition, analysis, and interpretation of data; revision of the manuscript; David Ley: conceptualization and design of the study; acquisition and interpretation of data; revision of the manuscript. All authors provided final approval of the version to be published.

\section{References}

1 Beca J, Gunn JK, Coleman L, Hope A, Reed PW, Hunt RW, et al. New white matter brain injury after infant heart surgery is associated with diagnostic group and the use of circulatory arrest. Circulation. 2013 Mar; 127(9): 971-9.

2 Dimitropoulos A, McQuillen PS, Sethi V, Moosa A, Chau V, Xu D, et al. Brain injury and development in newborns with critical congenital heart disease. Neurology. 2013 Jul; 81(3):241-8.

3 van Tilborg E, de Theije CG, van Hal M, Wagenaar N, de Vries LS, Benders MJ, et al. Origin and dynamics of oligodendrocytes in the developing brain: implications for perinatal white matter injury. Glia. 2018 Feb;66(2): 221-38.

4 Back SA, Rosenberg PA. Pathophysiology of glia in perinatal white matter injury. Glia. 2014 Nov;62(11):1790-815.

5 Ricci Z, Pezzella C, Romagnoli S, Iodice F, Haiberger R, Carotti A, et al. High levels of free haemoglobin in neonates and infants undergoing surgery on cardiopulmonary bypass. Interact Cardiovasc Thorac Surg. 2014 Aug;19(2):183-7.

6 Kagawa H, Morita K, Uno Y, Ko Y, Matsumura $\mathrm{Y}$, Kinouchi K, et al. Inflammatory response to hyperoxemic and normoxemic cardiopulmonary bypass in acyanotic pediatric patients. World J Pediatr Congenit Heart Surg. 2014 Oct;5(4):541-5.

7 Sheil ML, Luxford C, Davies MJ, Peat JK, Nunn G, Celermajer DS. Protein oxidation injury occurs during pediatric cardiopulmonary bypass. J Thorac Cardiovasc Surg. 2005 Oct;130(4):1054-61.

8 Schaer DJ, Vinchi F, Ingoglia G, Tolosano E, Buehler PW. Haptoglobin, hemopexin, and related defense pathways-basic science, clinical perspectives, and drug development. Front Physiol. 2014 Oct;5:415.

9 Olsson MG, Allhorn M, Bülow L, Hansson SR, Ley D, Olsson ML, et al. Pathological conditions involving extracellular hemoglobin: molecular mechanisms, clinical significance, and novel therapeutic opportunities for $\alpha(1)$ microglobulin. Antioxid Redox Signal. 2012 Sep;17(5):813-46.

10 Butt OI, Buehler PW, D’Agnillo F. Bloodbrain barrier disruption and oxidative stress in guinea pig after systemic exposure to modified cell-free hemoglobin. Am J Pathol. 2011 Mar;178(3):1316-28.

11 Ghosh S, Tan F, Ofori-Acquah SF. Spatiotemporal dysfunction of the vascular permeability barrier in transgenic mice with sickle cell disease. Anemia. 2012;2012:582018.

12 Favrais G, van de Looij Y, Fleiss B, Ramanantsoa N, Bonnin P, Stoltenburg-Didinger G, et al. Systemic inflammation disrupts the developmental program of white matter. Ann Neurol. 2011 Oct;70(4):550-65.

13 Pierre WC, Smith PL, Londono I, Chemtob S, Mallard C, Lodygensky GA. Neonatal microglia: the cornerstone of brain fate. Brain Behav Immun. 2017 Jan;59:333-45.

14 Reich B, Hoeber D, Bendix I, FelderhoffMueser U. Hyperoxia and the Immature Brain. Dev Neurosci. 2016;38(5):311-30.

15 Gerstner B, DeSilva TM, Genz K, Armstrong A, Brehmer F, Neve RL, et al. Hyperoxia causes maturation-dependent cell death in the developing white matter. J Neurosci. 2008 Jan;28(5):1236-45.

16 Brehmer F, Bendix I, Prager S, van de Looij Y, Reinboth BS, Zimmermanns J, et al. Interaction of inflammation and hyperoxia in a rat model of neonatal white matter damage. PLoS One. 2012;7(11):e49023.

17 Stinnett GR, Lin S, Korotcov AV, Korotcova L, Morton PD, Ramachandra SD, et al. Microstructural Alterations and Oligodendrocyte Dysmaturation in White Matter After Cardiopulmonary Bypass in a Juvenile Porcine Model. J Am Heart Assoc. 2017 Aug;6(8): e005997.

18 Ishibashi N, Scafidi J, Murata A, Korotcova L, Zurakowski D, Gallo V, et al. White matter protection in congenital heart surgery. Circulation. $2012 \mathrm{Feb}$; 125(7):859-71.

19 Jungner Å, Vallius S, Bruschettini M, Romantsik O, Gram M, Ley D. Cardiopulmonary bypass in the newborn: effects of circulatory cell-free hemoglobin and hyperoxia evaluated in a novel rat pup model. Intensive Care Med Exp. 2017 Oct;5(1):45.

20 Winterbourn CC. Oxidative reactions of hemoglobin. Methods Enzymol. 1990;186:265-72.

21 Basser PJ, Pierpaoli C. Microstructural and physiological features of tissues elucidated by quantitative-diffusion-tensor MRI. J Magn Reson B. 1996 Jun;111(3):209-19.

22 Nilsson M, Szczepankiewicz F, Lampinen B, Ahlgren A, P de Almeida Martins J, Lasic S, et al. An open-source framework for analysis of multidimensional diffusion MRI data implemented in MATLAB [abstract]. ISMRSESMRMB; 2018 June 16-21. Paris, France.

23 Klein HG. The red cell storage lesion(s): of dogs and men. Blood Transfus. 2017 Mar; 15(2):107-11.

24 Fleiss B, Tann CJ, Degos V, Sigaut S, Van Steenwinckel J, Schang AL, et al. Inflammation-induced sensitization of the brain in term infants. Dev Med Child Neurol. 2015 Apr;57 Suppl 3:17-28.
White Matter Development after Exposure to Cell-Free $\mathrm{Hb}$ and Hyperoxia
Dev Neurosci 2019;41:234-246

DOI: $10.1159 / 000505206$ 
25 Serdar M, Herz J, Kempe K, Lumpe K, Reinboth BS, Sizonenko SV, et al. Fingolimod protects against neonatal white matter damage and long-term cognitive deficits caused by hyperoxia. Brain Behav Immun. 2016 Feb;52: 106-19.

26 Lazic SE, Essioux L. Improving basic and translational science by accounting for litterto-litter variation in animal models. BMC Neurosci. 2013 Mar;14(1):37-11.

27 Agematsu K, Korotcova L, Scafidi J, Gallo V, Jonas RA, Ishibashi N. Effects of preoperative hypoxia on white matter injury associated with cardiopulmonary bypass in a rodent hypoxic and brain slice model. Pediatr Res. 2014 May;75(5):618-25.
28 Custódio CS, Mello BS, Filho AJ, de Carvalho Lima CN, Cordeiro RC, Miyajima F, et al. Neonatal Immune Challenge with Lipopolysaccharide Triggers Long-lasting Sex- and Age-related Behavioral and Immune/Neurotrophic Alterations in Mice: Relevance to Autism Spectrum Disorders. Mol Neurobiol. 2018 May;55(5):3775-88.

29 Burnsed JC, Chavez-Valdez R, Hossain MS, Kesavan K, Martin LJ, Zhang J, et al. Hypoxiaischemia and therapeutic hypothermia in the neonatal mouse brain - a longitudinal study. PLoS One. 2015 Mar;10(3):e0118889.

30 Bendix I, Hadamitzky M, Herz J, FelderhoffMüser U. Adverse neuropsychiatric development following perinatal brain injury: From a preclinical perspective. Pediatr Res. 2019 Jan; 85(2):198-215.

31 van Tilborg E, Achterberg EJ, van Kammen CM, van der Toorn A, Groenendaal F, Dijkhuizen RM, et al. Combined fetal inflammation and postnatal hypoxia causes myelin deficits and autism-like behavior in a rat model of diffuse white matter injury. Glia. 2018 Jan; 66(1):78-93.
32 Buehler PW, D'Agnillo F, Hoffman V, Alayash AI. Effects of endogenous ascorbate on oxidation, oxygenation, and toxicokinetics of cell-free modified hemoglobin after exchange transfusion in rat and guinea pig. J Pharmacol Exp Ther. 2007 Oct;323(1):49-60.

33 Semple BD, Blomgren K, Gimlin K, Ferriero DM, Noble-Haeusslein LJ. Brain development in rodents and humans: Identifying benchmarks of maturation and vulnerability to injury across species. Prog Neurobiol. 2013 Jul-Aug;106-107:1-16.

34 Jevtovic-Todorovic V, Absalom AR, Blomgren K, Brambrink A, Crosby G, Culley DJ, et al. Anaesthetic neurotoxicity and neuroplasticity: an expert group report and statement based on the BJA Salzburg Seminar. $\mathrm{Br}$ J Anaesth. 2013 Aug;111(2):143-51. 